\title{
Evolution of Two-Band Superfluidity from Weak to Strong Coupling
}

\author{
M. Iskin • C.A.R. Sá de Melo
}

Received: 3 May 2006 / Revised: 30 March 2007 / Published online: 4 July 2007

(C) Springer Science+Business Media, LLC 2007

\begin{abstract}
We analyze the evolution of two-band superfluidity from the weak to the strong coupling (Bardeen-Cooper-Schrieffer to Bose-Einstein condensation) limit. We find that population imbalances between the two bands can be created by tuning the intraband or interband (Josephson) interactions. In addition, when the Josephson interband interaction is tuned from negative to positive values, a quantum phase transition occurs from a 0 -phase to a $\pi$-phase state, depending on the relative phase of the two order parameters. We also find two undamped low energy collective excitations corresponding to in-phase phonon (Goldstone) and out-of-phase exciton (finite frequency) modes. Lastly, we derive the coupled Ginzburg-Landau equations, and show that they reduce to coupled Gross-Pitaevskii equations for two types of weakly interacting bosons (tightly bound fermions) in the BEC limit.
\end{abstract}

Keywords Two-band superconductivity $\cdot$ BCS-BEC crossover $\cdot$ Collective excitations · Time-dependent Ginzburg-Landau

PACS 03.75.Ss $\cdot$ 03.75. Hh $\cdot$ 05.30.Fk

\section{Introduction}

A two-band theory of superconductivity was introduced by Suhl et al. [1] in 1959 soon after the Bardeen-Cooper-Schrieffer (BCS) theory to allow for the possibility of multiple band crossings at the Fermi surface. This model has been applied to high- $T_{c}$ superconductors and $\mathrm{MgB}_{2}$, where, in the latter case, experimental properties can be well described by a two-band weak coupling BCS theory [2-5]. Unfortunately, interband or intraband interactions can not be tuned in these condensed matter

M. Iskin $(\varangle) \cdot$ C.A.R. Sá de Melo

School of Physics, Georgia Institute of Technology, Atlanta, GA 30332, USA

e-mail: gtg658i@mail.gatech.edu 
systems, and their properties can not be studied away from the BCS regime. However, two-band fermions may also be produced experimentally with ultracold atomic Fermi gases in optical lattices [6-8] or in single traps of several hyperfine states. In this case, (intraband and interband) interactions may be tuned using Feshbach resonances which allow for the study of the evolution of two-band superfluidity from the BCS to the Bose-Einstein condensation (BEC) limit. The BCS to BEC evolution in the two-band problem is much richer than the one-band case which has already been experimentally studied [6,9-15], since additional interaction parameters (interband and intraband) may be controlled externally.

Furthermore, a two-band model may be used not only to describe experiments involving several hyperfine states of the same fermion, but also may be used to study two different fermionic species (e.g. ${ }^{6} \mathrm{Li}$ and ${ }^{40} \mathrm{~K}$ ). In systems involving mixtures of two different alkali atoms, simple one-band theories may not be sufficient to describe the interactions between the two species of atoms, and two-band theories may be necessary to model future experiments. Thus, due to recent developments and advances in atomic physics described above, and in anticipation of future experiments, we describe here the BCS to BEC evolution of two-band superfluids for all coupling strengths at zero and finite temperatures.

The main results of our paper are as follows. We show that a quantum phase transition occurs from a 0 -phase to a $\pi$-phase state (depending on the relative phase of the order parameters of the two-bands) when the interband interaction $J$ is tuned from negative to positive values. We found that population imbalances between the two bands can be created by tuning intraband or interband interactions. In addition, we describe the evolution of two undamped low energy collective excitations corresponding to in-phase phonon (Goldstone) and out-of-phase exciton (finite frequency) modes. Near the critical temperature, we derive the coupled Ginzburg-Landau (GL) equations for a two-band superfluid, and show that they reduce to coupled GrossPitaevskii (GP) equations for two types of weakly interacting bosons (tightly bound fermions) in strong coupling.

The rest of the paper is organized as follows. We introduce the imaginary-time functional integration formalism in Sect. 2, and obtain the self-consistency (order parameter and number) equations. In Sect. 3, we discuss the evolution from BCS to BEC superfluidity at zero temperature, where we analyze the order parameter, chemical potential, population imbalance, and the low energy collective excitations. In Sect. 4, we present the evolution of superfluidity from the BCS to the BEC limit near the critical temperature, where we analyze the critical temperature, chemical potential, and derive the TDGL equation. A short summary of our conclusions is given in Sect. 5 .

\section{Effective Action}

To obtain the results mentioned above, we start from a multi-band Hamiltonian describing continuum superfluids with singlet pairing

$$
H=\sum_{n, \mathbf{k}, \sigma} \xi_{n, \sigma}(\mathbf{k}) a_{n, \sigma}^{\dagger}(\mathbf{k}) a_{n, \sigma}(\mathbf{k})-\sum_{n, m, r, s, \mathbf{q}} V_{n m}^{r s} b_{n m}^{\dagger}(\mathbf{q}) b_{r s}(\mathbf{q})
$$


Fig. 1 Schematic figure of the two bands with reference energies $\epsilon_{1,0}=0$ and $\epsilon_{2,0}=\epsilon_{D}$

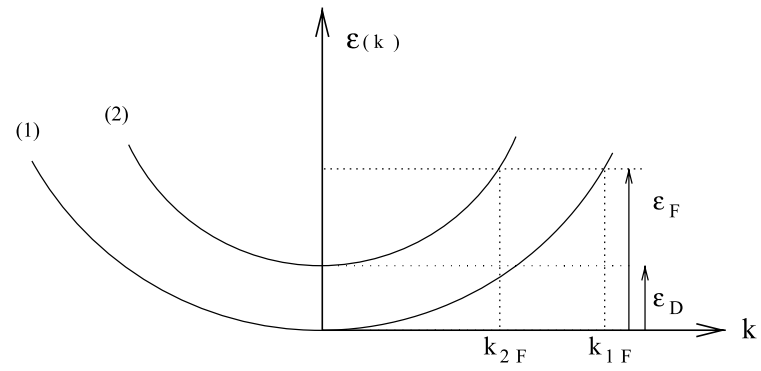

where $n, m, r$ and $s$ label different energy bands (or components), and $\sigma$ labels spins (or pseudo-spins). The operators $a_{n, \uparrow}^{\dagger}(\mathbf{k})$ and $b_{n m}^{\dagger}(\mathbf{q})=\sum_{\mathbf{k}} \Gamma_{n m}^{*}(\mathbf{k}) a_{n, \uparrow}^{\dagger}(\mathbf{k}+$ $\mathbf{q} / 2) a_{m, \downarrow}^{\dagger}(-\mathbf{k}+\mathbf{q} / 2)$ create a single and a pair of fermions, respectively. The symmetry factor $\Gamma_{n m}(\mathbf{k})$ characterizes the chosen angular momentum channel, where $\Gamma_{n m}(\mathbf{k})=k_{n m, 0} / \sqrt{k_{n m, 0}^{2}+k^{2}}$ is for the $s$-wave interaction in three dimensions [16]. Here, $k_{n m, 0} \sim R_{n m, 0}^{-1}$ sets the scale at small and large momenta, where $R_{n m, 0}$ plays the role of the interaction range. In addition $\xi_{n, \sigma}(\mathbf{k})=\epsilon_{n}(\mathbf{k})-\mu_{n, \sigma}$, where $\epsilon_{n}(\mathbf{k})=$ $\epsilon_{n, 0}+k^{2} /\left(2 M_{n}\right)$ is the kinetic energy $(\hbar=1)$ and $M_{n}$ is the band mass of the fermions.

From now on, we focus on a two-band system such that $V_{n m}^{r s}=V_{n r} \delta_{n m} \delta_{r s}$ with distinct intraband $\left(V_{11}, V_{22}\right)>0$ and interband $\left(V_{12}=V_{21}=J\right)$ interactions. Notice that, $J$ plays the role of the Josephson interaction which couples the two energy bands. In addition, we assume that the total number of fermions is fixed ( $N=N_{1}+$ $N_{2}$ ) such that the chemical potentials of fermions are identical $\left(\mu_{n, \sigma}=\mu\right)$, and that the reference energies are such that $\epsilon_{1,0}=0$ and $\epsilon_{2,0}=\epsilon_{D}>0$, as shown in Fig. 1. Here, $\epsilon_{D} \leq \epsilon_{F}=k_{1, F}^{2} /\left(2 M_{1}\right)$, where $\epsilon_{F}$ is the Fermi energy and $k_{n, F}$ are the Fermi momenta $k_{1, F}=k_{F}$ and $k_{2, F}=\left[2 M_{2}\left(\epsilon_{F}-\epsilon_{D}\right)\right]^{1 / 2}$. Since the low energy physics depends weakly on $k_{n, 0}$ in dilute systems $\left(\mathcal{N} R_{0}^{3} \ll 1\right)$ characterized by $k_{n, 0}^{3} \gg k_{n, F}^{3}$ where $\mathcal{N}=N / \mathcal{V}$ is the density of fermions and $\mathcal{V}$ is the volume, we assume for simplicity that $k_{n, 0}=k_{0} \gg k_{F}$.

The Gaussian action for the Hamiltonian $H$ is (in units of $k_{B}=1, \beta=1 / T$ ) [17]

$$
S_{\text {gauss }}=S_{0}+\frac{\beta}{2} \sum_{q} \Lambda^{\dagger}(-q) \mathbf{F}^{-1}(q) \Lambda(q),
$$

where $q=\left(\mathbf{q}, i v_{\ell}\right)$ denotes both momentum and bosonic Matsubara frequency $v_{\ell}=$ $2 \ell \pi / \beta$. Here, the vector $\Lambda^{\dagger}(-q)$ is the order parameter fluctuation field, and the matrix $\mathbf{F}^{-1}(q)$ is the inverse fluctuation propagator. The saddle point action is

$$
S_{0}=\sum_{n, \mathbf{k}}\left\{\beta\left[\xi_{n}(\mathbf{k})-E_{n}(\mathbf{k})\right]-2 \ln \left[1+e^{-\beta E_{n}(\mathbf{k})}\right]\right\}-\beta \sum_{n, m} g_{n m} \Delta_{n, 0}^{*} \Delta_{m, 0},
$$

where $E_{n}(\mathbf{k})=\left[\xi_{n}^{2}(\mathbf{k})+\left|\Delta_{n}(\mathbf{k})\right|^{2}\right]^{\frac{1}{2}}$ is the energy of the quasiparticles and $\Delta_{n}(\mathbf{k})=$ $\Delta_{n, 0} \Gamma_{n}(\mathbf{k})$ is the order parameter. Here, the matrix elements of $\mathbf{g}$ is associated with 
Fig. 2 (Color on-line) Schematic phase diagram of 0 -phase and $\pi$-phase states

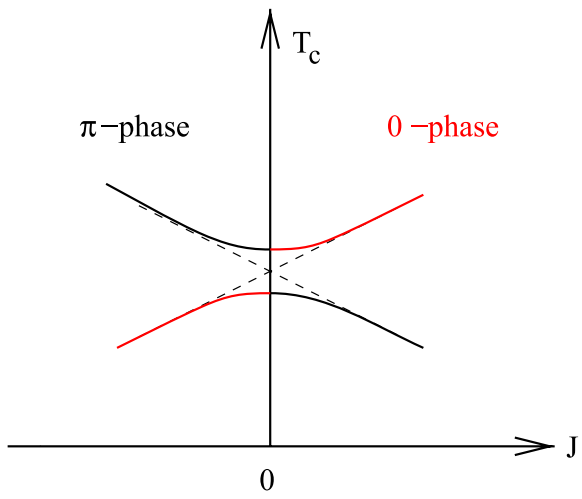

the inverse interaction matrix $\mathbf{V}$, and are given by

$$
\left(\begin{array}{ll}
g_{11} & g_{12} \\
g_{21} & g_{22}
\end{array}\right)=\frac{1}{\operatorname{det} \mathbf{V}}\left(\begin{array}{cc}
-V_{22} & J \\
J & -V_{11}
\end{array}\right),
$$

where det $\mathbf{V}=V_{11} V_{22}-J^{2}>0$.

The action leads to the thermodynamic potential $\Omega_{\text {gauss }}=\Omega_{0}+\Omega_{\text {fluct }}$, where $\Omega_{0}=$ $S_{0} / \beta$ is the saddle point and $\Omega_{\text {fluct }}=(1 / \beta) \sum_{q} \ln \operatorname{det}\left[\mathbf{F}^{-1}(q) /(2 \beta)\right]$ is the fluctuation contribution to $\Omega_{\text {gauss }}$. Expressing $\Delta_{n, 0}$ in terms of its amplitude and phase

$$
\Delta_{n, 0}=\left|\Delta_{n, 0}\right| \exp \left(i \varphi_{n}\right)
$$

shows explicity the Josephson coupling energy $\left[V_{22}\left|\Delta_{1,0}\right|^{2}+V_{11}\left|\Delta_{2,0}\right|^{2}\right.$ $\left.-2 J\left|\Delta_{1,0} \Delta_{2,0}\right| \cos \left(\varphi_{2}-\varphi_{1}\right)\right] / \operatorname{det} \mathbf{V}$ of $\Omega_{0}$. When $J>0$, only the 0 -phase (or in phase) $\varphi_{2}=\varphi_{1}$ solution is stable. However, when $J<0$, only the $\pi$-phase (or out of phase) $\varphi_{2}=\varphi_{1}+\pi$ solution is stable. Thus, a phase transition occurs from the 0 -phase to the $\pi$-phase when the sign of $J$ is tuned from negative to positive values as shown in Fig. 2.

From the stationary condition $\partial S_{0} / \partial \Delta_{n}^{*}(q)=0$, we obtain the order parameter equation

$$
\left(\begin{array}{ll}
O_{11} & O_{12} \\
O_{21} & O_{22}
\end{array}\right)\left(\begin{array}{l}
\Delta_{1,0} \\
\Delta_{2,0}
\end{array}\right)=0,
$$

where the matrix elements are given by $O_{n m}=-g_{n m}-\delta_{n m} \sum_{\mathbf{k}}\left|\Gamma_{m}(\mathbf{k})\right|^{2} \tanh [\beta$ $\left.\times E_{m}(\mathbf{k}) / 2\right] /\left[2 E_{m}(\mathbf{k})\right]$. Here, $\delta_{n m}$ is the Kronecker delta. Notice that the order parameter amplitudes are the same for both the 0 -phase and $\pi$-phase as can be shown directly from (6), but their relative phases are either 0 or $\pi$. In what follows, we analyse only the 0-phase state, keeping in mind that analogous results (with appropriate relative phase changes) apply to the $\pi$-phase state. We can elliminate $V_{n n}$ in favor of scattering length $a_{n n}$ via the relation

$$
\frac{1}{V_{n n}}=-\frac{M_{n} \mathcal{V}}{4 \pi a_{n n}}+\sum_{\mathbf{k}} \frac{\left|\Gamma_{n}(\mathbf{k})\right|^{2}}{2 \epsilon_{n}(\mathbf{k})},
$$

which can be solved to obtain $1 /\left(k_{n, F} a_{n n}\right)=k_{0} / k_{n, F}-4 \pi /\left(k_{n, F} V_{n n} M_{n} \mathcal{V}\right)$. 
The order parameter equation needs to be solved self-consistently with the number equation $N=-\partial \Omega / \partial \mu$ leading to $N_{\text {gauss }}=N_{0}+N_{\text {fluct }}$, and is given by

$$
N_{\text {gauss }}=\sum_{\mathbf{k}, \sigma, m} \mathcal{N}_{0, m}(\mathbf{k})-\frac{1}{\beta} \sum_{q} \frac{\partial\left[\operatorname{det} \mathbf{F}^{-1}(q)\right] / \partial \mu}{\operatorname{det} \mathbf{F}^{-1}(q)} .
$$

Here, the first term is the saddle point $\left(N_{1}+N_{2}\right)$ and the second term is the fluctuation $\left(N_{\text {fluct }}\right)$ contribution, where $\mathcal{N}_{0, m}=1 / 2-\xi_{m}(\mathbf{k}) \tanh \left[\beta E_{m}(\mathbf{k}) / 2\right] /\left[2 E_{m}(\mathbf{k})\right]$ is the momentum distribution. The inclusion of $N_{\text {fluct }}$ is very important near the critical temperature, however, $N_{0}$ may be sufficient at low temperatures $[18,19]$. Thus, we discuss next the $T=0$ case.

\section{Zero Temperature}

In this section, we analyze the saddle point order parameter amplitudes $\left|\Delta_{n, 0}\right|$ and the chemical potential $\mu$ at $T=0$. In the strictly BCS and BEC limits, the self-consistent (order parameter and number) equations are decoupled, and play reversed roles. In the BCS (BEC) limit, the order parameter equations determine $\left|\Delta_{n, 0}\right|(\mu)$, and the number equations determine $\mu\left(\left|\Delta_{n, 0}\right|\right)$, which is discussed next.

The BCS limit is characterized by positive chemical potential with respect to the bottom of the fermion band $\mu>0$ and $\max \left\{\left|\Delta_{1,0}\right|,\left|\Delta_{2,0}\right|\right\} \ll \epsilon_{F}$, where pairing occurs between the fermions whose energy are close to $\epsilon_{F}$. In this limit, the solutions of the order parameter equation are $\max \left\{\left|\Delta_{1,0}\right|,\left|\Delta_{2,0}\right|\right\} \sim 8 \epsilon_{F} \exp \left[-2+\pi k_{0} /\left(2 k_{F}\right)-\right.$ $\left.\phi_{-}\right]$for the larger of the order parameter amplitudes and $\min \left\{\left|\Delta_{1,0}\right|,\left|\Delta_{2,0}\right|\right\} \sim$ $8 \epsilon_{F} \exp \left[-2+\pi k_{0} /\left(2 k_{F}\right)-\phi_{+}\right]$for the smaller of the order parameter amplitudes, while the number equation leads to $\mu \approx \epsilon_{F}$. Here, $\phi_{ \pm}=\lambda_{+} \pm\left[\lambda_{+}^{2}-1 / \operatorname{det} \lambda\right]^{1 / 2}$ where $\lambda_{ \pm}=\left(\lambda_{11} \pm \lambda_{22}\right) /(2 \operatorname{det} \lambda)$, $\operatorname{det} \lambda=\lambda_{11} \lambda_{22}-\lambda_{12} \lambda_{21}$, and $\lambda_{n m}=V_{n m} D_{m}$ are the dimensionless interaction parameters with $D_{m}=M_{m} \mathcal{V} k_{m, F} /\left(2 \pi^{2}\right)$ is the density of states per spin at the Fermi energy. The familiar one-band results are recovered when $J \rightarrow 0$ upon the use of the relation between $V_{n n}$ and $a_{n n}$.

On the other hand, the BEC limit is characterized by negative chemical potential with respect to the bottom of the fermion band $\mu<0$ and $\max \left\{\left|\Delta_{1,0}\right|,\left|\Delta_{2,0}\right|\right\} \ll$ $|\mu| \ll \epsilon_{0}$, where pairing occurs between all fermions. In this limit, the solution of the order parameter equations is $\mu=-\epsilon_{0}\left[\pi k_{0} /\left(2 k_{F} \phi_{+}\right)-1\right]^{2}$, while the number equation leads to $\left|\Delta_{m, 0}\right|^{2}=\left(8 \pi \mathcal{N}_{m} / M_{m}\right) \sqrt{|\mu| /\left(2 M_{m}\right)}$. Here, $\epsilon_{0}=k_{0}^{2} /\left(2 M_{1}\right)$ and $\mathcal{N}_{m}=N_{m} / \mathcal{V}$. Notice that the total density of fermions is $\mathcal{N}=\mathcal{N}_{1}+\mathcal{N}_{2}=\left(k_{1, F}^{3}+\right.$ $\left.k_{2, F}^{3}\right) /\left(3 \pi^{2}\right)$. The familiar one-band results are again recovered when $J \rightarrow 0$ upon the use of the relation between $V_{n n}$ and $a_{n n}$.

Next, we analyze numerically (by solving coupled self-consistency equations) the evolution of the order parameter amplitudes and the chemical potential from the BCS to the BEC limit for identical bands $\left(M_{1}=M_{2}=M\right)$ with zero offset $\left(\epsilon_{D}=0\right)$ at zero temperature. For this purpose, we set $k_{0} \approx 256 k_{F}$ and $V_{22}=0.001$ in units of $5.78 /\left(M k_{F} \mathcal{V}\right)$ [or $1 /\left(k_{F} a_{22}\right) \approx-3.38$ ], and analyze two cases.

In the first case, we solve for $\mu,\left|\Delta_{1,0}\right|$ and $\left|\Delta_{2,0}\right|$ as a function of $V_{11}$ [or $\left.1 /\left(k_{F} a_{11}\right)\right]$, and show $\Delta_{n, 0}$ in Fig. 3 for fixed values of $J$. The unitarity limit is reached at $V_{11} \approx 1.0132 V_{22}$ [or $1 /\left(k_{F}\left|a_{11}\right|\right) \approx 0$ ] while $\mu$ changes sign at $V_{11} \approx$ 
Fig. 3 Plots of order parameter amplitude $\left|\Delta_{n, 0}\right|$ (in units of $\left.\epsilon_{F}\right)$, and versus $V_{11}$ [in units of $\left.5.78 /\left(M k_{F} \mathcal{V}\right)\right]$ and versus $1 /\left(k_{F} a_{11}\right)$ for $J=0.001 V_{22}$ (hollow squares) and $J=0.0001 V_{22}$ (solid squares) (a)

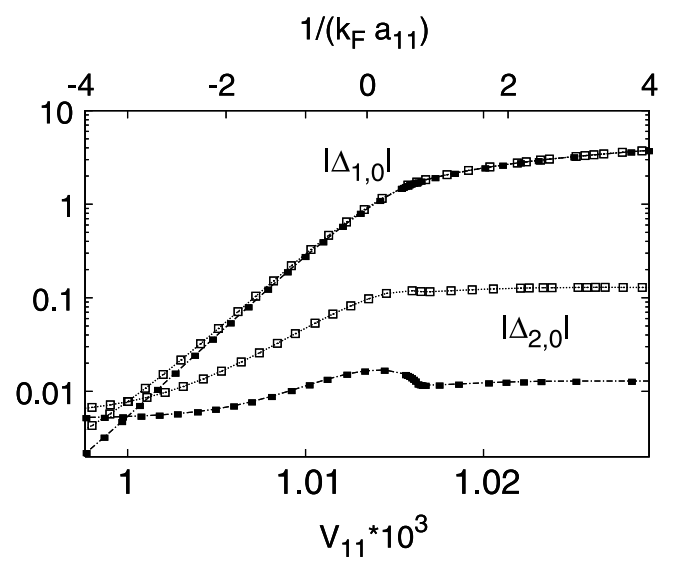

(b)

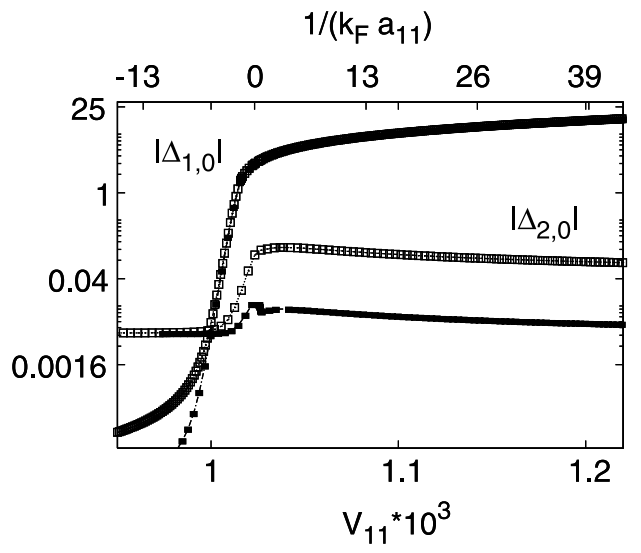

$1.0159 V_{22}$ [or $\left.1 /\left(k_{F} a_{11}\right) \approx 0.69\right]$. The evolution of $\left|\Delta_{1,0}\right|$ is similar to the one band result [18] where it grows monotonically with increasing $V_{11}$. However, the evolution of $\left|\Delta_{2,0}\right|$ is non-monotonic where it has a hump approximately $V_{11} \approx 1.0155 V_{22}$ [or $1 /\left(k_{F} a_{11}\right) \approx 0.58$ ], and it decreases for stronger interactions until it vanishes (not shown). In the case of ultra-cold atoms, the order parameter may be measured using similar techniques as for one band systems [15] involving only ${ }^{6} \mathrm{Li}$. In Fig. 4, we show that both bands have similar populations for $V_{11} \sim V_{22}$. However, as $V_{11} / V_{22}$ increases, fermions from the second band are transferred to the first, where bound states are easily formed and reduce the free energy. In coupled two-band systems, spontaneous population imbalances are induced by the increasing scattering parameter (interaction) in contrast with the one band case, where population imbalances are prepared externally [20, 21].

In the second case, we solve for $\mu,\left|\Delta_{1,0}\right|$ and $\left|\Delta_{2,0}\right|$ as a function of $J$, and show $\mu$ in Fig. 5 and band populations $N_{n}$ in Fig. 6 for fixed values of $V_{11} / V_{22}$. The order parameters $\left|\Delta_{1,0}\right|$ and $\left|\Delta_{2,0}\right|$ grow with increasing $J$ (not shown). Notice the 
Fig. 4 Plots of fraction of fermions $N_{n} / N$ versus $V_{11}$ [in units of $\left.5.78 /\left(M k_{F} \mathcal{V}\right)\right]$ and versus $1 /\left(k_{F} a_{11}\right)$ for $J=0.001 V_{22}$ (hollow squares) and $J=0.0001 V_{22}$ (solid squares)

Fig. 5 Plots of chemical potential $\mu$ (in units of $\epsilon_{F}$ ) versus $J$ [in units of $\left.5.78 /\left(M k_{F} \mathcal{V}\right)\right]$ for $V_{11}=\gamma V_{22}$ where $\gamma=1$ (dotted lines), 1.010 (solid squares), 1.014 (hollow squares), 1.016 (crossed lines) and 1.030 (solid lines); or $1 /\left(k_{F} a_{11}\right) \approx-3.38,-0.81$, $0.20,0.70$ and 4.17 , respectively
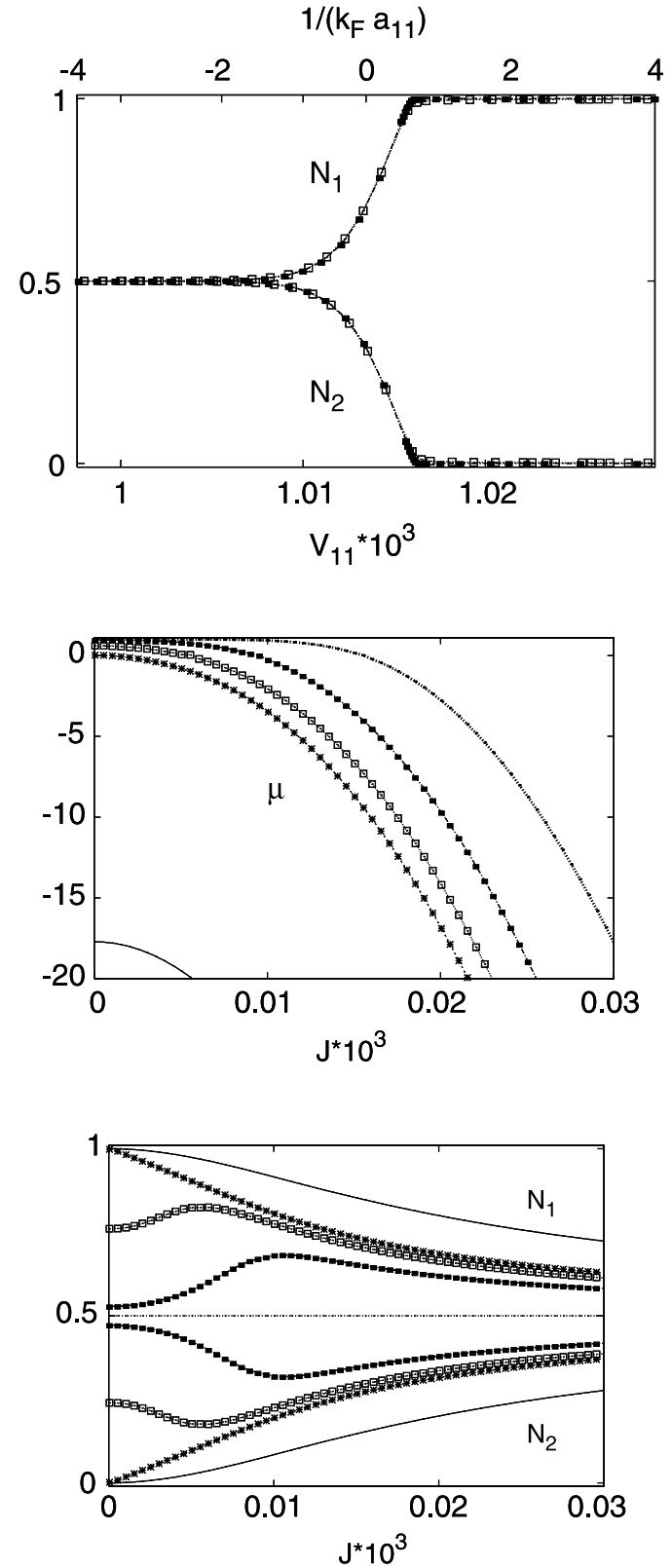

Fig. 6 Plots of fraction of fermions $N_{n} / N$ versus $J$ [in units of $\left.5.78 /\left(M k_{F} \mathcal{V}\right)\right]$ for $V_{11}=\gamma V_{22}$ where $\gamma=1$ (dotted lines), 1.010 (solid squares), 1.014 (hollow squares), 1.016 (crossed lines) and 1.030 (solid lines); or $1 /\left(k_{F} a_{11}\right) \approx-3.38,-0.81$, $0.20,0.70$ and 4.17 , respectively 
Next, we discuss the low energy collective excitations at $T=0$, which are determined by the poles of the propagator matrix $\mathbf{F}(q)$ for the pair fluctuation fields $\Lambda_{n}(q)$, which describe the Gaussian deviations about the saddle point order parameter. The poles of $\mathbf{F}(q)$ are determined by the condition $\operatorname{det} \mathbf{F}^{-1}(q)=0$, which leads to collective (amplitude and phase) modes, when the usual analytic continuation $i v_{\ell} \rightarrow w+i 0^{+}$is performed. The easiest way to get the phase collective mode is to integrate out the amplitude field to obtain a phase-only effective action. Notice that, a well defined low frequency expansion is possible only at $T=0$ for $s$-wave systems, due to Landau damping present at finite temperatures causing the collective modes to decay into the two quasiparticle continuum.

The phase-only collective excitations in the BCS and BEC limits lead to a Goldstone mode $w^{2}(\mathbf{q})=v^{2}|\mathbf{q}|^{2}$ characterized by the speed of sound

$$
v^{2}=\frac{D_{1} v_{1}^{2}+D_{2} v_{2}^{2}}{D_{1}+D_{2}} ;
$$

and a finite frequency mode $w^{2}(\mathbf{q})=w_{0}^{2}+u^{2}|\mathbf{q}|^{2}$ characterized by the finite frequency and the speed

$$
\begin{aligned}
& w_{0}^{2}=4 \alpha\left|g_{12} \Delta_{1,0} \Delta_{2,0}\right| \sqrt{\frac{\epsilon_{F}}{|\mu|}} \frac{D_{1}+D_{2}}{D_{1} D_{2}}, \\
& u^{2}=\frac{D_{1} v_{2}^{2}+D_{2} v_{1}^{2}}{D_{1}+D_{2}} .
\end{aligned}
$$

In the BCS limit, $\alpha=1$ and $v_{n}=v_{n, F} / \sqrt{3}$, while $\alpha=1 / \pi$ and $v_{n}=\left|\Delta_{n, 0}\right| / \sqrt{8 M_{n}|\mu|}$ in the BEC limit. Here, $v_{n, F}$ is the Fermi velocity. Notice that, the trivial limit of oneband (say only band 1 exists) is directly recovered by taking $J \rightarrow 0$ and $D_{2}=v_{2}=0$, which leads to $v=v_{1}, w_{0}=0$ and $u=0$. It is also illustrative to analyse the eigenvectors associated with these solutions in the BCS and BEC limits. In the limit of $\mathbf{q} \rightarrow \mathbf{0}$, we obtain $\left(\theta_{1}, \theta_{2}\right) \propto\left(\left|\Delta_{1,0}\right|,\left|\Delta_{2,0}\right|\right)$ for the Goldstone mode corresponding to an in-phase solution, while $\left(\theta_{1}, \theta_{2}\right) \propto\left(D_{2}\left|\Delta_{1,0}\right|,-D_{1}\left|\Delta_{2,0}\right|\right)$, for the finite frequency (exciton) mode corresponding to an out-of-phase solution. Notice that, in the case of identical bands with zero off-set and identical order parameter amplitudes, the collective modes simplifies to a perfectly in-phase where $\left(\theta_{1}, \theta_{2}\right) \propto(1,1)$, and a perfectly out-of-phase modes where $\left(\theta_{1}, \theta_{2}\right) \propto(1,-1)$. These in-phase and out-ofphase collective modes are associated with the in-phase and out-of-phase fluctuations of the order parameters around their saddle point values, respectively. Our findings generalize Leggett's BCS results [17, 22].

\section{Finite Temperatures}

Next, we discuss two band superfluidity near the critical temperature $T_{c}$, where $\left|\Delta_{1,0}\right| \sim\left|\Delta_{2,0}\right| \rightarrow 0$. Our basic motivation here is to investigate the low frequency and long wavelength behavior of the order parameter near $T_{c}$. For $T=T_{c}$, the order 
parameter equation reduces to

$$
\operatorname{det} \mathbf{O}=O_{11} O_{22}-O_{12} O_{21}=0
$$

and the saddle point number equation $N_{0}=\sum_{\mathbf{k}, n} n_{F}\left[\xi_{n}(\mathbf{k})\right]$ corresponds to the number of unbound fermions, where $n_{F}(x)=1 /[\exp (\beta x)+1]$ is the Fermi distribution. While $N_{0}$ is sufficient in the BCS limit, the inclusion of $N_{\text {fluct }}$ is crucial in the BEC limit to produce the qualitatively correct physics, and can be obtained as follows.

Near $T=T_{c}$, the fluctuation action $S_{\text {fluct }}$ reduces to $S_{\text {fluct }}=\beta \sum_{q, n, m} L_{n m}^{-1}(q)$ $\times \Lambda_{n}^{*}(q) \Lambda_{m}(q)$ where

$$
L_{n n}^{-1}=-g_{n n}-\sum_{\mathbf{k}} \frac{1-n_{F}\left(\xi_{n+}\right)-n_{F}\left(\xi_{n-}\right)}{\xi_{n+}+\xi_{n-}-i v_{\ell}}\left|\Gamma_{n}(\mathbf{k})\right|^{2}
$$

corresponds to the fluctuation propagator of band $n, L_{n \neq m}^{-1}(q)=g_{n m}$, and $\xi_{n \pm}=$ $\xi_{n}(\mathbf{k} \pm \mathbf{q} / 2)$. Thus, the resulting action leads to $\Omega_{\text {fluct }}=(1 / \beta) \sum_{q} \ln \left[\operatorname{det} \mathbf{L}^{-1}(q) / \beta^{2}\right]$, where $\operatorname{det} \mathbf{L}^{-1}(q)=L_{11}^{-1}(q) L_{22}^{-1}(q)-g_{12} g_{21}$. Notice that, $\operatorname{det} \mathbf{L}^{-1}(0)=0$ also produces (12), which is the Thouless condition. After the analytic continuation $i v_{\ell} \rightarrow$ $w+i 0^{+}$, we expand $L_{n n}^{-1}(q)$ to first order in $w$ and second order in $\mathbf{q}$ such that

$$
L_{n n}^{-1}(q)=a_{n}+\sum_{i, j} \frac{c_{n}^{i j}}{2 M_{n}} q_{i} q_{j}-d_{n} w .
$$

All static and dynamic expansion coefficients are presented in the Appendix. Notice that, $c_{n}^{i j}=c_{n} \delta_{i j}$ is isotropic for the $s$-wave considered here. In addition, the time-dependent coefficient $d_{n}$ is a complex number, and its imaginary part reflects the decay of Cooper pairs into the two-particle continuum for $\mu>0$. However, for $\mu<0$, the imaginary part of $d_{n}$ vanishes and the behavior of the order parameter is propagating reflecting the presence of stable bound states.

In order to obtain $\Omega_{\text {fluct }}$, there are two contributions, one from the scattering states and the other from poles of $\mathbf{L}(q)$. The pole contribution dominates in the BEC limit. In this case, we evaluate $\operatorname{det} \mathbf{L}^{-1}(q)=0$ and find the poles

$$
w_{ \pm}(\mathbf{q})=A_{+}+B_{+}|\mathbf{q}|^{2} \pm \sqrt{\left(A_{-}+B_{-}|\mathbf{q}|^{2}\right)^{2}+\frac{g_{12} g_{21}}{d_{1} d_{2}}},
$$

where $A_{ \pm}=\left(a_{1} d_{2} \pm a_{2} d_{1}\right) /\left(2 d_{1} d_{2}\right)$ and $B_{ \pm}=\left(M_{2} d_{2} c_{1} \pm M_{1} d_{1} c_{2}\right) /\left(4 M_{1} M_{2} d_{1} d_{2}\right)$. Notice that, when $J \rightarrow 0$, we recover the limit of uncoupled bands with $w_{n}(\mathbf{q})=$ $a_{n} / d_{n}+|\mathbf{q}|^{2} c_{n} /\left(2 M_{n} d_{n}\right)$. It is also illustrative to analyze the eigenvectors associated with these poles. In the $\mathbf{q} \rightarrow \mathbf{0}$ limit, the eigenvectors $\left[\Lambda_{1}^{\dagger}(0), \Lambda_{2}^{\dagger}(0)\right]=$ $\left[g_{12}, a_{1}-d_{1} w_{ \pm}(0)\right]$ correspond to an in-phase mode for $w_{+}(\mathbf{q})$ and an out-ofphase mode for $w_{-}(\mathbf{q})$ when $J>0$, however, they correspond to an out-of-phase mode for $w_{+}(\mathbf{q})$ and an in-phase mode for $w_{-}(\mathbf{q})$ when $J<0$. Thus, we obtain $\Omega_{\text {fluct }}=(1 / \beta) \sum_{ \pm, q} \ln \left[\beta\left(i v_{\ell}-w(\mathbf{q})\right)\right]$, which leads to

$$
N_{\text {fluct }}=\sum_{ \pm, \mathbf{q}} \frac{\partial w(\mathbf{q})}{\partial \mu} n_{B}[w(\mathbf{q})]
$$


In the BEC limit, $\partial w_{ \pm}(\mathbf{q}) / \partial \mu=2$, and the poles can also be written as $w_{ \pm}(\mathbf{q})=$ $-\mu_{B, \pm}+|\mathbf{q}|^{2} /\left(2 M_{B, \pm}\right)$, where $\mu_{B, \pm}$ is the chemical potential and $M_{B, \pm}$ is the mass of the corresponding bosons. In the case of identical bands with zero offset, $c_{1}=c_{2}=$ $c$ and $d_{1}=d_{2}=d, \mu_{B, \pm}=-\left[a_{1}+a_{2} \pm \sqrt{\left(a_{1}-a_{2}\right)^{2}+4 g_{12} g_{21}}\right] /(2 d)$ and $M_{B, \pm}=$ $2 M$ in the BEC limit. Notice that the + bosons always condense first for any $J$ (independent of its sign) since $\mu_{B,+} \rightarrow 0$ first. Next, we analyze $T_{c}$ in the strict BCS and BEC limits, where self-consistency equations are uncoupled allowing analytical results.

The BCS limit is characterized by positive chemical potential with respect to the bottom of the particle band $\mu>0$ and $\mu \approx \epsilon_{F}$. In this limit, solutions to the order parameter equation (12) are $T_{c, \mp}=\left(8 \epsilon_{F} / \pi\right) \exp \left[\gamma-2+\pi k_{0} /\left(2 k_{F}\right)-\phi_{ \pm}\right]$, while the number equation (8) leads to $\mu \approx \epsilon_{F}$.

On the other hand, the BEC limit is characterized by negative chemical potential with respect to the particle band $\mu<0$ and $\epsilon_{0} \gg|\mu| \gg T_{c}$. In this limit, the solution of the order parameter equation is $\mu=-\epsilon_{0}\left[\pi k_{0} /\left(2 k_{F} \phi_{+}\right)-1\right]^{2}$, while the number equation $\mathcal{N} / 2=\mathcal{N}_{B,+}+\mathcal{N}_{B,-}$ with $\mathcal{N}_{B,+} \gg \mathcal{N}_{B,-}$ leads to $T_{c,+}=$ $\left(2 \pi / M_{B,+}\right)\left[\mathcal{N}_{B,+} / \zeta(3 / 2)\right]^{2 / 3}$, since the + bosons condense first. Notice that, the physical critical temperature is $T_{c}=\max \left\{T_{c,+}, T_{c,-}\right\}=T_{c,+}$ for any $J$. Therefore, $T_{c}$ grows continuously from an exponential dependence on interaction to a constant BEC temperature.

Next, we obtain the time-dependent Ginzburg-Landau (TDGL) equations for $T \approx T_{c}$. To study the evolution of the TDGL functional near $T_{c}$, we need to expand the effective action $S_{\text {eff }}$ to fourth order in $\Lambda_{n}(x)$ around the saddle point order parameter $\left|\Delta_{n}\right| \rightarrow 0$ leading to

$$
\left[a_{n}+b_{n}\left|\Lambda_{n}(x)\right|^{2}-\frac{c_{n}}{2 M_{n}} \nabla^{2}-i d_{n} \frac{\partial}{\partial t}\right] \Lambda_{n}(x)+g_{n \neq m} \Lambda_{m}(x)=0,
$$

in the real space $x=(\mathbf{x}, t)$ representation. The coefficient $b_{n}$ of the nonlinear term (given in the Appendix) is always positive and guarantees the stability of the theory. In the BCS limit, (17) reduces to the coupled GL equations of two BCS type superconductors. However, in the BEC limit, it is more illustrative to derive TDGL equations in the rotated basis of + and - bosons $\left(\Phi_{+}^{\dagger}, \Phi_{-}^{\dagger}\right)=\left(\Lambda_{1}^{\dagger}, \Lambda_{2}^{\dagger}\right) \mathbf{R}^{\dagger}$, where $\mathbf{R}$ is the unitary matrix that diagonalizes the linear part of the TDGL equations. In this basis, (17) reduces to generalized GP equations of $\Phi_{+}$and $\Phi_{-}$bosons showing explicitly terms coming from density-density interactions such as $U_{ \pm \pm}\left|\Phi_{ \pm}\right|^{2} \Phi_{ \pm}$or $U_{ \pm \mp}\left|\Phi_{ \pm}\right|^{2} \Phi_{\mp}$. In the case of identical bands with zero offset, this leads to $U_{++}=U_{--}=b /\left(2 d^{2}\right)$ and $U_{+-}=U_{-+}=3 \mathrm{~b} /\left(2 d^{2}\right)$ as the repulsive density-density interactions.

\section{Conclusions}

We studied the evolution of two-band superfluidity from the BCS to the BEC limit as a function of interaction strength and scattering parameter, where we analyzed superfluid properties of a dilute Fermi gas at zero temperature $(T=0)$ in the ground state and near critical temperatures $\left(T=T_{c}\right)$. The main results of our paper are as follows. 
At zero temperature, we showed that a quantum phase transition occurs from a 0 -phase to a $\pi$-phase state depending on the relative phase of the two order parameters, when the interband interaction is tuned from negative to positive values. We found that population imbalances between the two bands can be created by tuning intraband or interband interactions. In addition, we described the evolution of two undamped low energy collective excitations corresponding to in-phase phonon (Goldstone) and out-of-phase exciton (finite frequency) modes.

Near the critical temperature, we derived the coupled time-dependent GinzburgLandau (TDGL) functional near $T_{c}$. We recovered the usual TDGL equations for BCS superfluids in the BCS limit, whereas in the BEC limit we recovered the coupled Gross-Pitaevskii equations for two types of weakly interacting bosons (tightly bound fermions). We also showed that the Cooper pairs have short lifetimes in the BCS limit decaying into the two particle continuum when chemical potential is above the bottom of the particle band $(\mu>0)$, however "Cooper molecules" (bound states) have long lifetimes in the BEC limit when chemical potential is below the bottom of the particle band $(\mu<0)$.

To conclude, the main contribution of our manuscript is to analyze the BCS to BEC evolution of two-band superfluids, which is potentially relevant to experiments with multi-component ultracold Fermi gases in optical lattices or continuum (trapped) systems. In the presence of additional (interband and intraband) interaction parameters, two-band theories rather than one-band theories may be necessary to describe future experiments involving ultracold fermions.

Acknowledgements We thank NSF (DMR-0304380) for support.

\section{Appendix}

In this appendix, we perform a small $\mathbf{q}$ and $i v_{\ell} \rightarrow w+i 0^{+}$expansion near $T_{c}$, where we assumed that the fluctuation field $\Lambda_{n}(\mathbf{x}, t)$ is a slowly varying function of $\mathbf{x}$ and $t$. The zeroth order coefficient $L_{n n}^{-1}(0,0)$ is given by

$$
a_{n}=-g_{n n}-\sum_{\mathbf{k}} \frac{X_{n}}{2 \xi_{n}(\mathbf{k})}\left|\Gamma_{n}(\mathbf{k})\right|^{2},
$$

where $X_{n}=\tanh \left[\beta \xi_{n}(\mathbf{k}) / 2\right]$. The second order coefficient $M_{n} \partial^{2} L_{n n}^{-1}(\mathbf{q}, 0) /\left(\partial q_{i} \partial q_{j}\right)$ evaluated at $\mathbf{q}=\mathbf{0}$ is given by

$$
c_{n}^{i j}=\sum_{\mathbf{k}}\left\{\left[\frac{X_{n}}{8 \xi_{n}^{2}(\mathbf{k})}-\frac{\beta Y_{n}}{16 \xi_{n}(\mathbf{k})}\right] \delta_{i j}+\frac{\beta^{2} X_{n} Y_{n}}{16 M_{n} \xi_{n}(\mathbf{k})} k_{i} k_{j}\right\}\left|\Gamma_{n}(\mathbf{k})\right|^{2},
$$

where $Y_{n}=\operatorname{sech}^{2}\left[\beta \xi_{n}(\mathbf{k}) / 2\right]$ and $\delta_{i j}$ is the Kronecker delta. The coefficient of the fourth order term is approximated at $\mathbf{q}=\mathbf{0}$, and given by

$$
b_{n}=\sum_{\mathbf{k}}\left[\frac{X_{n}}{4 \xi_{n}^{3}(\mathbf{k})}-\frac{\beta Y_{n}}{8 \xi_{n}^{2}(\mathbf{k})}\right]\left|\Gamma_{n}(\mathbf{k})\right|^{4}
$$


The time-dependent coefficient has real and imaginary parts, and for the $s$-wave case is given by

$$
d_{n}=\sum_{\mathbf{k}} \frac{X_{n}}{4 \xi_{n}^{2}(\mathbf{k})}\left|\Gamma_{n}(\mathbf{k})\right|^{2}+i \frac{\beta \pi D_{n} \epsilon_{0}}{8\left(\epsilon_{0}+\mu\right)} \sqrt{\frac{\mu}{\epsilon_{F}}} \Theta(\mu),
$$

where $\Theta(x)$ is the Heaviside function.

For completeness, we present the asymptotic forms of $a_{n}, b_{n}, c_{n}$ and $d_{n}$. The BCS limit is characterized by positive chemical potential with respect to the bottom of the particle band $\mu>0$ and $\mu \approx \epsilon_{F}$. In this limit, we find $a_{n}=-g_{n n}+D_{n}\left[\ln \left(T / T_{c}\right)+\right.$ $\left.\phi_{-}\right], b_{n}=7 D_{n} \zeta(3) /\left(8 T_{c}^{2} \pi^{2}\right), c_{n}=7 \epsilon_{F} D_{n} \zeta(3) /\left(12 T_{c}^{2} \pi^{2}\right)$, and $d_{n}=D_{n}\left[1 /\left(4 \epsilon_{F}\right)+\right.$ $\left.i /\left(8 T_{c}\right)\right]$, where $\zeta(x)$ is the Zeta function, and $T_{c}$ is the physical critical temperature.

The BEC limit is characterized by negative chemical potential with respect to the particle band $\mu<0$ and $\epsilon_{0} \gg|\mu| \gg T_{c}$. In this limit, we find $a_{n}=-g_{n n}-$ $\pi D_{n} \epsilon_{0} /\left[2 \sqrt{\epsilon_{F}}\left(\sqrt{|\mu|}+\sqrt{\epsilon_{0}}\right)\right], b_{n}=\pi D_{n} /\left(4|\mu| \sqrt{2|\mu| \epsilon_{F}}\right), c_{n}=\pi D_{n} /\left(16 \sqrt{|\mu| \epsilon_{F}}\right)$ and $d_{n}=\pi D_{n} /\left(8 \sqrt{|\mu| \epsilon_{F}}\right)$.

\section{References}

1. H. Suhl, B.T. Matthias, L.R. Walker, Phys. Rev. Lett. 3, 552 (1959)

2. M. Iavarone, G. Karapetrov, A.E. Koshelev, W.K. Kwok, G.W. Crabtree, D.G. Hinks, W.N. Kang, E.-M. Choi, H.J. Kim, H.-J. Kim, S.I. Lee, Phys. Rev. Lett. 89, 187002 (2001)

3. F. Bouquet, Y. Wang, I. Sheikin, T. Plackowski, A. Junod, S. Lee, S. Tajima, Phys. Rev. Lett. 89, 257001 (2002)

4. S. Tsuda, T. Yokoya, Y. Takano, H. Kito, A. Matsushita, F. Yin, J. Itoh, H. Harima, S. Shin, Phys. Rev. Lett. 91, 127001 (2003)

5. J. Geerk, R. Schneider, G. Linker, A.G. Zaitsev, R. Heid, K.-P. Bohnen, H.V. Löhneysen, Phys. Rev. Lett. 94, 227005 (2005)

6. M. Köhl, H. Moritz, T. Stöferle, K. Günter, T. Esslinger, Phys. Rev. Lett. 94, 080403 (2005)

7. S. Ospelkaus, C. Ospelkaus, L. Humbert, K. Sengstock, K. Bongs, Phys. Rev. Lett. 97, 120403 (2006)

8. J.K. Chin, D.E. Miller, Y. Liu, C. Stan, W. Setiawan, C. Sanner, K. Xu, W. Ketterle, Nature 443, 961 (2006)

9. K.E. Strecker, G.B. Partridge, R.G. Hulet, Phys. Rev. Lett. 91, 080406 (2003)

10. C.A. Regal, M. Greiner, D.S. Jin, Phys. Rev. Lett. 92, 040403 (2004)

11. M. Bartenstein, A. Altmeyer, S. Riedl, S. Jochim, C. Chin, J.H. Denschlag, R. Grimm, Phys. Rev. Lett. 92, 120401 (2004)

12. J. Kinast, S.L. Hemmer, M.E. Gehm, A. Turlapov, J.E. Thomas, Phys. Rev. Lett. 92, 150402 (2004)

13. T. Bourdel, L. Khaykovich, J. Cubizolles, J. Zhang, F. Chevy, M. Teichmann, L. Tarruell, S.J.J.M.F. Kokkelmans, C. Salomon, Phys. Rev. Lett. 93, 050401 (2004)

14. M.W. Zwierlein, J.R. Abo-Shaeer, A. Schirotzek, C.H. Schunck, W. Ketterle, Nature 435, 1047 (2005)

15. G.B. Partridge, K.E. Strecker, R.I. Kamar, M.W. Jack, R.G. Hulet, Phys. Rev. Lett. 95, 020404 (2005)

16. M. Iskin, C.A.R. Sá de Melo, Phys. Rev. A 74, 013608 (2006)

17. M. Iskin, C.A.R. Sá de Melo, Phys. Rev. B 72, 024512 (2005)

18. C.A.R. Sá de Melo, M. Randeria, J.R. Engelbrecht, Phys. Rev. Lett. 71, 3202 (1993)

19. J.R. Engelbrecht, M. Randeria, C.A.R. Sá de Melo, Phys. Rev. B 55, 15153 (1997)

20. M.W. Zwierlein, A. Schirotzek, C.H. Schunck, W. Ketterle, Science 311, 492 (2006)

21. G.B. Partridge, W. Lui, R.I. Kamar, Y. Liao, R.G. Hulet, Science 311, 503 (2006)

22. A.J. Leggett, Progr. Theor. Phys. 36, 901 (1966) 